\title{
Molecular Dynamics Study of Adsorption of the Lennard-Jones Truncated and Shifted Fluid on Planar Walls
}

Michaela Heier, ${ }^{\dagger}$ Felix Diewald, ${ }^{\ddagger}$ Martin T. Horsch, ${ }^{\dagger} \uparrow$ Kai Langenbach, ${ }^{* \dagger}{ }^{\dagger}$ Ralf Müller, ${ }^{\ddagger}$ and Hans Hasse ${ }^{\dagger}$

$\dagger$ Laboratory of Engineering Thermodynamics, Technische Universität Kaiserslautern, Kaiserslautern, Germany

$\ddagger$ Institute of Applied Mechanics, Technische Universität Kaiserslautern, Kaiserslautern, Germany

ICurrent address: Scientific Computing Department, STFC Daresbury Laboratory, UK Research and Innovation, Warrington, United Kingdom

E-mail: kai.langenbach@mv-uni-kl.de

Phone: +49 (0)631 2054685. Fax: +49 (0)631 2053835

\section{Supporting Information}

\section{Simulation Scenario}

The simulation scenario contains an atomistic wall which is fixed at the bottom of the simulation box by a LJTS-9-3 potential with a cutoff radius of $2.5 \sigma_{\mathrm{f}}$. This potential is located at a height of $y=0 \sigma_{\mathrm{f}}$ and is defined as: 


$$
\begin{gathered}
u_{\mathrm{LJ} 93}(r)=\frac{4}{3} \pi \varepsilon_{\mathrm{SS}} \rho_{\mathrm{s}} \sigma_{\mathrm{sS}}^{3}\left[\frac{1}{15}\left(\frac{\sigma_{\mathrm{ss}}}{r}\right)^{9}-\frac{1}{2}\left(\frac{\sigma_{\mathrm{ss}}}{r}\right)^{3}\right] \\
u_{\mathrm{LJTS} 93}(r)=\left\{\begin{array}{ll}
u_{\mathrm{LJ} 93}(r)-u_{\mathrm{LJ} 93}\left(r_{\mathrm{c}}\right) & r \leq r_{\mathrm{c}} \\
0 & r>r_{\mathrm{c}}
\end{array} .\right.
\end{gathered}
$$

Where $\varepsilon_{\mathrm{ss}}$ and $\sigma_{\mathrm{ss}}$ are the energy and size parameter of the solid-solid (ss) interaction and $\rho_{\mathrm{s}}$ the density of the atomistic wall. Due to its cutoff radius, this potential is acting only on the atomistic wall because the height of the atomistic wall is always bigger than $2.5 \sigma_{\mathrm{f}}$. At the top of the simulation box (at a height of $y=55 \sigma_{\mathrm{f}}$ ) a membrane is applied. It avoids layer growth underneath the atomistic wall due to periodic boundary conditions. Especially, when investigating adsorption at high pressures, a strong layer growth would occur at both sides of the wall. This would lead to a reduction of the amount of bulk vapor phase. The membrane avoids this layer growth by applying a reset force of $F=-20 \cdot \Delta y$, whereas $\Delta y$ is the distance from the membrane to particles above the membrane.

\section{Size Effects}

To examine the influence of the amount of fluid particles $N_{\mathrm{f}}$ on the surface excess, simulations for several sets of parameters are performed. In these simulations the number of fluid particles and therefore the system size in $x$ - and $z$-direction is changed. The simulations are carried out for a constant fluid density $\rho_{\mathrm{f}}$. Table 1 gives an overview of the simulation conditions for which the size effect was studied.

Table 1: Overview of simulation conditions for studying the size effect.

\begin{tabular}{c|c|c}
\hline$T / \varepsilon_{\mathrm{f}} k^{-1}$ & $\rho_{\mathrm{s}} / \sigma_{\mathrm{f}}^{-3}$ & $\zeta$ \\
\hline 0.7 & 1.07 & 0.35 \\
& & 0.65 \\
\hline 0.8 & 1.07 & 0.35 \\
& & 0.65 \\
\hline
\end{tabular}


The number of fluid particles varies between $N_{\mathrm{f}}=1,931$ and 9,155 . The results of these simulations are shown in Figure 1 and Table 2.

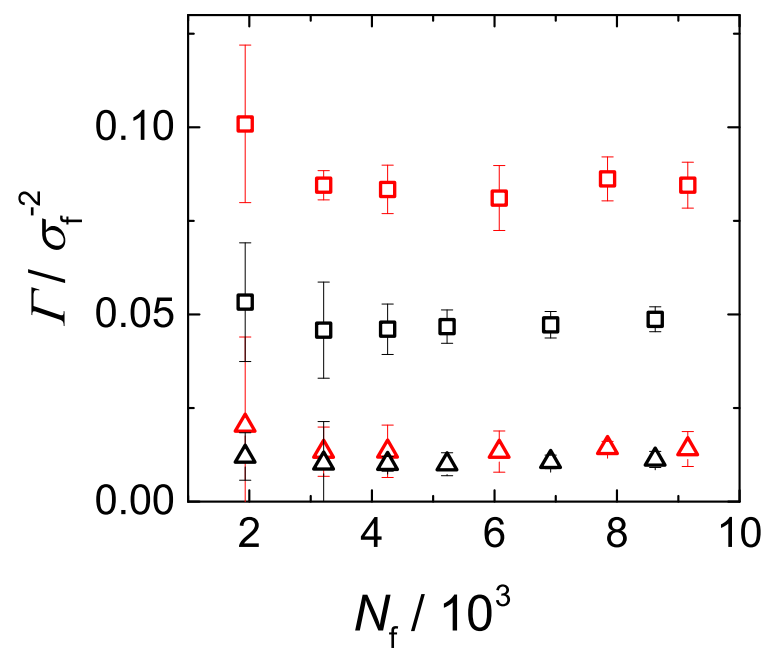

Figure 1: Surface excess $\Gamma$ as a function of the number of fluid particles $N_{\mathrm{f}}$ for temperature $T=0.7 \varepsilon_{\mathrm{f}} / k$ (red) and $T=0.8 \varepsilon_{\mathrm{f}} / k$ (black), and reduced solid-fluid interaction energy $\zeta=0.35(\triangle)$ and $\zeta=0.65(\square)$. 
Table 2: Simulation results for testing the influence of the number of particles $N_{\mathrm{f}}$ on the surface excess $\Gamma$ for $T=0.7,0.8$, and $0.9 \varepsilon_{\mathrm{f}} / k$ and $\zeta=0.35$ and 0.65 . The number in parentheses indicates the statistical uncertainty in the last decimal digit.

\begin{tabular}{|c|c|c|c|}
\hline$\overline{T / \varepsilon_{\mathrm{f}}} k^{-1}$ & $\zeta$ & $\Gamma / \sigma_{\mathrm{f}}^{-2}$ & $N_{\mathrm{f}}$ \\
\hline \multirow[t]{12}{*}{0.7} & 0.35 & $0.018(12)$ & 1931 \\
\hline & & $0.015(5)$ & 3213 \\
\hline & & $0.0146(47)$ & 4255 \\
\hline & & $0.0139(31)$ & 6078 \\
\hline & & $0.0143(20)$ & 7846 \\
\hline & & $0.0142(34)$ & 9155 \\
\hline & 0.65 & $0.095(25)$ & 1931 \\
\hline & & $0.085(9)$ & 3213 \\
\hline & & $0.084(7)$ & 4255 \\
\hline & & $0.083(13)$ & 6078 \\
\hline & & $0.086(5)$ & 7846 \\
\hline & & $0.085(5)$ & 9155 \\
\hline \multirow[t]{12}{*}{0.8} & 0.35 & $0.013(13)$ & 1931 \\
\hline & & $0.011(6)$ & 3213 \\
\hline & & $0.0097(46)$ & 4255 \\
\hline & & $0.009(6)$ & 5227 \\
\hline & & $0.0106(37)$ & 6916 \\
\hline & & $0.0116(37)$ & 8622 \\
\hline & 0.65 & $0.057(20)$ & 1931 \\
\hline & & $0.0427(45)$ & 3213 \\
\hline & & $0.0446(42)$ & 4255 \\
\hline & & $0.046(6)$ & 5227 \\
\hline & & $0.047(5)$ & 6916 \\
\hline & & $0.0485(47)$ & 8622 \\
\hline
\end{tabular}

For $N_{\mathrm{f}}<6,000$ finite size effects occur. Above $N_{\mathrm{f}}=6,000$ the surface excess stays constant within the uncertainties.

\section{Numerical simulation results}

All results of the adsorption simulations for the surface excess and the adsorption layer thickness are given in Table 3 . 
Table 3: Bulk fluid density $\rho_{\mathrm{f}}^{\mathrm{b}}$, bulk fluid pressure $p_{\mathrm{f}}^{\mathrm{b}}$, surface excess $\Gamma$, layer thickness $\delta$, and number of adsorption layer peaks $N_{\text {peaks }}$ for the adsorption simulations for changing temperature $T$, solid density $\rho_{\mathrm{s}}$, and reduced solid-fluid interaction energy $\zeta$. The number in parentheses indicates the statistical uncertainty in the last decimal digit.

\begin{tabular}{|c|c|c|c|c|c|c|c|}
\hline$T / T_{\mathrm{c}}$ & $\rho_{\mathrm{s}} / \sigma_{\mathrm{f}}^{-3}$ & $\zeta$ & $\rho_{\mathrm{f}}^{\mathrm{b}} / \sigma_{\mathrm{f}}^{-3}$ & $p_{\mathrm{f}}^{\mathrm{b}} / \varepsilon_{\mathrm{f}} \sigma_{\mathrm{f}}^{-3}$ & $\Gamma / \sigma_{\mathrm{f}}^{-2}$ & $\delta / \sigma_{\mathrm{f}}$ & $N_{\text {peaks }}$ \\
\hline \multirow[t]{30}{*}{0.7} & \multirow[t]{30}{*}{1.07} & \multirow[t]{10}{*}{0.35} & $0.003243(14)$ & $0.002394(39)$ & $0.0043(7)$ & $1.51(23)$ & 1 \\
\hline & & & $0.006404(30)$ & $0.004611(34)$ & $0.0080(15)$ & $1.57(35)$ & 1 \\
\hline & & & $0.00971(5)$ & $0.00683(11)$ & $0.0122(23)$ & $1.70(23)$ & 1 \\
\hline & & & $0.01281(14)$ & $0.00876(18)$ & $0.018(7)$ & $1.9(6)$ & 1 \\
\hline & & & $0.01609(10)$ & $0.01068(33)$ & $0.023(8)$ & $1.92(42)$ & 1 \\
\hline & & & $0.01894(14)$ & $0.01226(27)$ & $0.026(12)$ & $2.0(5)$ & 2 \\
\hline & & & $0.02241(24)$ & $0.01408(32)$ & $0.031(12)$ & $2.04(35)$ & 2 \\
\hline & & & $0.02574(37)$ & $0.0159(9)$ & $0.037(18)$ & $2.1(8)$ & 2 \\
\hline & & & $0.02814(14)$ & $0.0167(7)$ & $0.048(11)$ & $2.5(6)$ & 2 \\
\hline & & & $0.0310(6)$ & $0.01787(36)$ & $0.057(30)$ & $3.0(31)$ & 2 \\
\hline & & \multirow[t]{8}{*}{0.514} & $0.003146(20)$ & $0.002323(39)$ & $0.0090(10)$ & $1.66(23)$ & 1 \\
\hline & & & $0.00618(10)$ & $0.00446(9)$ & $0.019(5)$ & $1.85(48)$ & 1 \\
\hline & & & $0.00934(10)$ & $0.00659(12)$ & $0.0298(48)$ & $2.04(35)$ & 1 \\
\hline & & & $0.01229(8)^{\prime}$ & $0.00847(11)$ & $0.042(6)$ & $2.14(23)$ & 1 \\
\hline & & & $0.01540(18)$ & $0.01023(41)$ & $0.056(19)$ & $2.23(52)$ & 2 \\
\hline & & & $0.01801(29)$ & $0.01176(25)$ & $0.071(12)$ & $2.39(30)$ & 2 \\
\hline & & & $0.02111(28)$ & $0.01342(40)$ & $0.090(14)$ & $2.6(7)$ & 2 \\
\hline & & & $0.02354(17)$ & $0.0146(8)$ & $0.145(9)$ & $4.3(14)$ & 2 \\
\hline & & \multirow[t]{7}{*}{0.65} & $0.002991(36)$ & $0.002213(31)$ & $0.0167(19)$ & $1.85(38)$ & 1 \\
\hline & & & $0.00585(6)$ & $0.00423(9)$ & $0.0356(36)$ & $2.07(30)$ & 1 \\
\hline & & & $0.00874(23)$ & $0.00618(22)$ & $0.060(11)$ & $2.3(6)$ & 1 \\
\hline & & & $0.01140(12)$ & $0.00791(16)$ & $0.0870(45)$ & $2.42(46)$ & 1 \\
\hline & & & $0.01404(14)$ & $0.00951(13)$ & $0.125(11)^{\prime}$ & $2.80(48)$ & 2 \\
\hline & & & $0.01618(28)$ & $0.01073(11)$ & $0.164(13)$ & $2.92(38)$ & 2 \\
\hline & & & $0.01743(49)$ & $0.01147(22)$ & $0.272(25)$ & $4.2(26)$ & 2 \\
\hline & & \multirow[t]{5}{*}{0.75} & $0.00284(8)$ & $0.00211(7)$ & $0.0239(40)$ & $1.92(30)$ & 1 \\
\hline & & & $0.00800(11)$ & $0.00570(14)$ & $0.097(7)$ & $2.4(7)$ & 1 \\
\hline & & & $0.01199(40)$ & $0.00820(29)$ & $0.225(19)$ & $2.95(23)$ & 2 \\
\hline & & & $0.0139(5)$ & $0.00942(42)$ & $0.446(25)$ & $3.62(35)$ & 2 \\
\hline & & & $0.0140(13)$ & $0.0094(8)$ & $0.74(6)$ & $4.6(6)$ & 3 \\
\hline \multirow[t]{13}{*}{0.77} & \multirow[t]{13}{*}{1.07} & \multirow[t]{13}{*}{0.75} & $0.002968(40)$ & $0.002424(45)$ & $0.0178(20)$ & $1.79(19)$ & 1 \\
\hline & & & $0.00866(10)$ & $0.00680(12)$ & $0.0632(47)$ & $2.17(38)$ & 1 \\
\hline & & & 0.01391(19) & $0.01045(23)$ & $0.130(10)^{\prime}$ & $2.6(6)$ & 2 \\
\hline & & & $0.0185(5)$ & $0.0136(6)$ & $0.219(25)$ & $3.90(38)$ & 2 \\
\hline & & & $0.02025(45)$ & $0.0147(6)$ & $0.270(23)$ & $3.1(6)$ & 2 \\
\hline & & & $0.02191(41)$ & $0.01568(14)$ & $0.351(19)$ & $3.43(48)$ & 2 \\
\hline & & & $0.02384(31)$ & $0.01689(34)$ & $0.416(15)$ & $3.7(7)$ & 2 \\
\hline & & & $0.02403(39)$ & $0.01691(37)$ & $0.503(20)$ & $3.96(30)$ & 3 \\
\hline & & & $0.02506(33)$ & $0.01749(36)$ & $0.616(17)$ & $4.3(6)$ & 3 \\
\hline & & & $0.0255(6)$ & $0.0180(10)$ & $0.748(32)$ & $4.5(6)$ & 3 \\
\hline & & & $0.0258(13)$ & $0.0179(12)$ & $0.90(6)$ & $5.0(11)$ & 3 \\
\hline & & & $0.0265(15)$ & $0.0185(14)$ & $1.05(7)$ & $5.2(7)$ & 3 \\
\hline & & & $0.0269(14)$ & $0.0187(9)$ & $1.16(7)$ & $5.6(6)$ & 3 \\
\hline 0.8 & 1.07 & 0.35 & $0.003263(20)$ & $0.002770(37)$ & $0.0032(10)$ & $1.51(23)$ & 1 \\
\hline
\end{tabular}




\begin{tabular}{|c|c|c|c|c|c|}
\hline & $\begin{array}{c}0.00976(9) \\
0.01622(16) \\
0.02253(20) \\
0.02850(16) \\
0.03362(46) \\
0.0399(5) \\
0.0467(8) \\
0.0531(6)\end{array}$ & $\begin{array}{c}0.00798(13) \\
0.01262(35) \\
0.01688(28) \\
0.0205(9) \\
0.0234(7) \\
0.0269(5) \\
0.02955(36) \\
0.0320(8)\end{array}$ & $\begin{array}{c}0.0092(41) \\
0.015(11) \\
0.023(10) \\
0.030(13) \\
0.033(24) \\
0.041(27) \\
0.057(39) \\
0.064(23)\end{array}$ & $\begin{array}{c}1.54(38) \\
1.7(6) \\
1.85(48) \\
2.0(6) \\
1.9(7) \\
2.1(8) \\
2.8(21) \\
2.6(7)\end{array}$ & $\begin{array}{l}1 \\
1 \\
2 \\
2 \\
2 \\
2 \\
2 \\
2\end{array}$ \\
\hline 0.514 & $\begin{array}{c}0.003193(15) \\
0.00952(9) \\
0.01582(17) \\
0.02196(14) \\
0.02755(27) \\
0.03251(45) \\
0.0382(6) \\
0.0447(10) \\
0.0502(14)\end{array}$ & $\begin{array}{c}0.002709(33) \\
0.00780(16) \\
0.01237(31) \\
0.01654(36) \\
0.0200(5) \\
0.0227(5) \\
0.0257(12) \\
0.0284(6) \\
0.0309(11)\end{array}$ & $\begin{array}{c}0.0067(7) \\
0.021(4) \\
0.035(10) \\
0.051(11) \\
0.078(16) \\
0.086(23) \\
0.123(28) \\
0.16(5) \\
0.20(6)\end{array}$ & $\begin{array}{c}1.57(19) \\
1.79(19) \\
1.9(6) \\
2.11(46) \\
2.45(48) \\
2.5(9) \\
3.0(8) \\
3.2(13) \\
4.2(43)\end{array}$ & $\begin{array}{l}1 \\
1 \\
1 \\
2 \\
2 \\
2 \\
2 \\
2 \\
2\end{array}$ \\
\hline 0.65 & $\begin{array}{c}0.003099(22) \\
0.00919(12) \\
0.01522(14) \\
0.02091(12) \\
0.0261(6) \\
0.0300(9) \\
0.0351(9) \\
0.0389(16)\end{array}$ & $\begin{array}{c}0.002632(47) \\
0.00754(12) \\
0.01187(42) \\
0.01587(42) \\
0.0191(6) \\
0.0213(8) \\
0.0242(9) \\
0.0261(7)\end{array}$ & $\begin{array}{c}0.0113(11) \\
0.037(7) \\
0.065(9) \\
0.102(14) \\
0.149(30) \\
0.212(41) \\
0.277(43) \\
0.44(8)\end{array}$ & $\begin{array}{c}1.70(23) \\
1.98(23) \\
2.20(35) \\
2.45(48) \\
2.8(6) \\
3.2(8) \\
3.40(48) \\
4.2(9)\end{array}$ & $\begin{array}{l}1 \\
1 \\
2 \\
2 \\
2 \\
2 \\
2 \\
3\end{array}$ \\
\hline 0.75 & $\begin{array}{c}0.002998(27) \\
0.00883(14) \\
0.01438(26) \\
0.01948(27) \\
0.02157(28) \\
0.02379(17) \\
0.02594(48) \\
0.02701(46) \\
0.0286(7) \\
0.0299(6) \\
0.03168(35) \\
0.0319(14) \\
0.0331(8) \\
0.0329(9) \\
\end{array}$ & $\begin{array}{c}0.00255(42) \\
0.00725(18) \\
0.01133(39) \\
0.0149(7) \\
0.0163(5) \\
0.01770(33) \\
0.01898(47) \\
0.01967(34) \\
0.02056(32) \\
0.0216(6) \\
0.0224(8) \\
0.0224(10) \\
0.0231(13) \\
0.0231(9) \\
\end{array}$ & $\begin{array}{c}0.0162(14) \\
0.055(7) \\
0.106(12) \\
0.170(14) \\
0.205(14) \\
0.258(8) \\
0.312(24) \\
0.355(20) \\
0.438(38) \\
0.529(32) \\
0.610(17) \\
0.78(7) \\
0.849(39) \\
1.052(46) \\
\end{array}$ & $\begin{array}{c}1.79(19) \\
2.11(35) \\
2.39(30) \\
2.67(35) \\
2.89(19) \\
3.11(38) \\
3.3(8) \\
3.5(6) \\
3.8(10) \\
4.2(13) \\
4,3(8) \\
4.8(9) \\
4.9(10) \\
5.5(6) \\
\end{array}$ & $\begin{array}{l}1 \\
1 \\
2 \\
2 \\
2 \\
2 \\
2 \\
2 \\
3 \\
3 \\
3 \\
3 \\
3 \\
3 \\
\end{array}$ \\
\hline 1.0 & $\begin{array}{c}0.00253(7) \\
0.00703(9) \\
0.01055(39) \\
0.01373(17) \\
0.0162(5) \\
0.0186(10) \\
0.0218(6) \\
0.0240(7) \\
0.0270(12) \\
0.0284(11) \\
0.0316(17) \\
\end{array}$ & $\begin{array}{c}0.00215(6) \\
0.00584(18) \\
0.00855(38) \\
0.01088(35) \\
0.01263(30) \\
0.01436(47) \\
0.0165(5) \\
0.0178(8) \\
0.0198(13) \\
0.0206(6) \\
0.0223(13) \\
\end{array}$ & $\begin{array}{c}0.0394(34) \\
0.1439(39) \\
0.296(20) \\
0.457(12) \\
0.638(30) \\
0.771(48) \\
0.931(30) \\
1.175(33) \\
1.34(6) \\
1.59(5) \\
2.23(9) \\
\end{array}$ & $\begin{array}{c}1.95(35) \\
2.33(23) \\
2.77(48) \\
3.1(6) \\
3.6(7) \\
3.9(6) \\
4.34(48) \\
5.00(38) \\
5.3(10) \\
5.9(9) \\
7.3(13) \\
\end{array}$ & $\begin{array}{l}1 \\
2 \\
2 \\
2 \\
2 \\
2 \\
3 \\
3 \\
3 \\
4 \\
4 \\
\end{array}$ \\
\hline 1.5 & $0.000784(40)$ & $0.000674(39)$ & $0.1265(20)$ & $2.39(30)$ & 1 \\
\hline
\end{tabular}




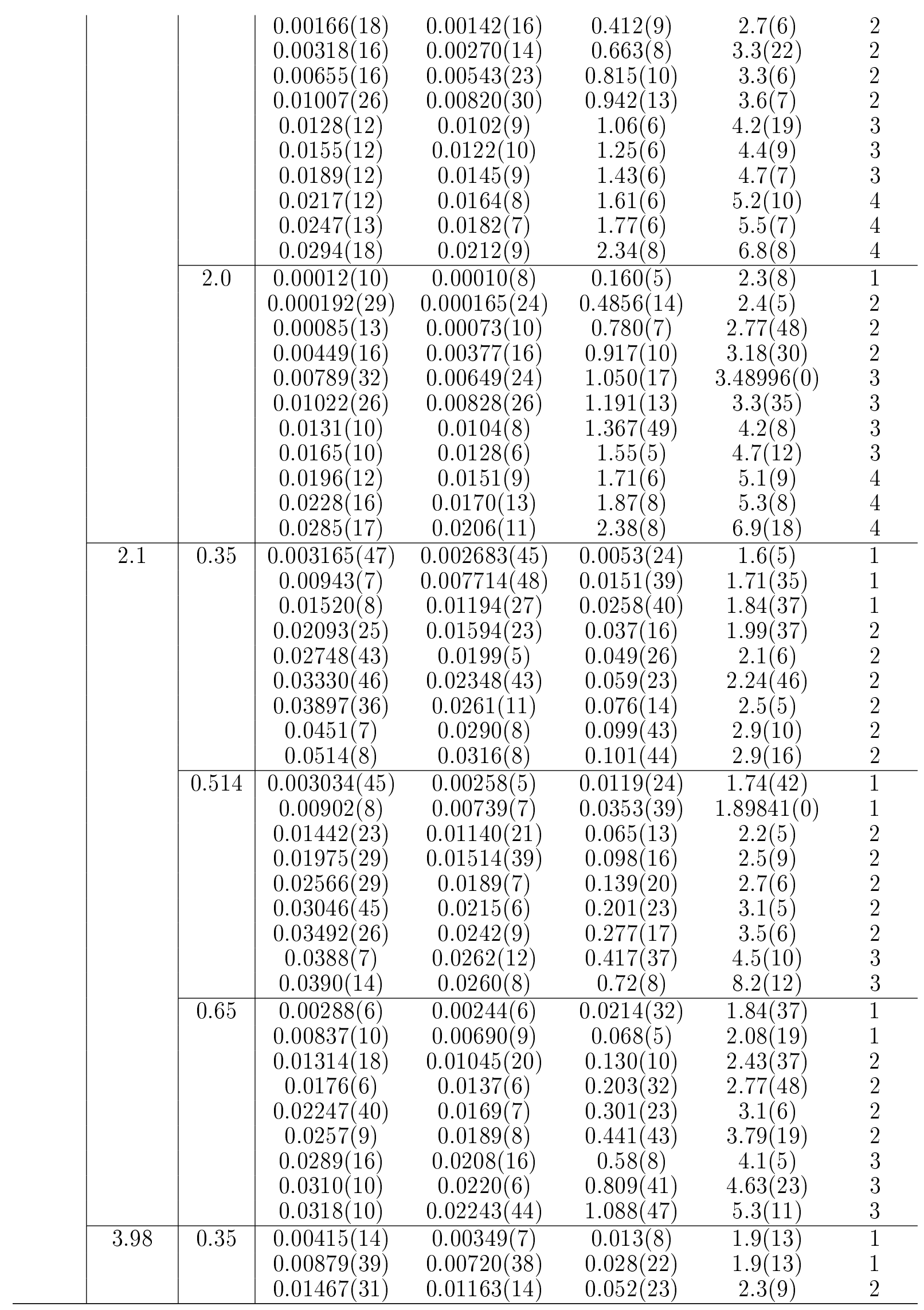




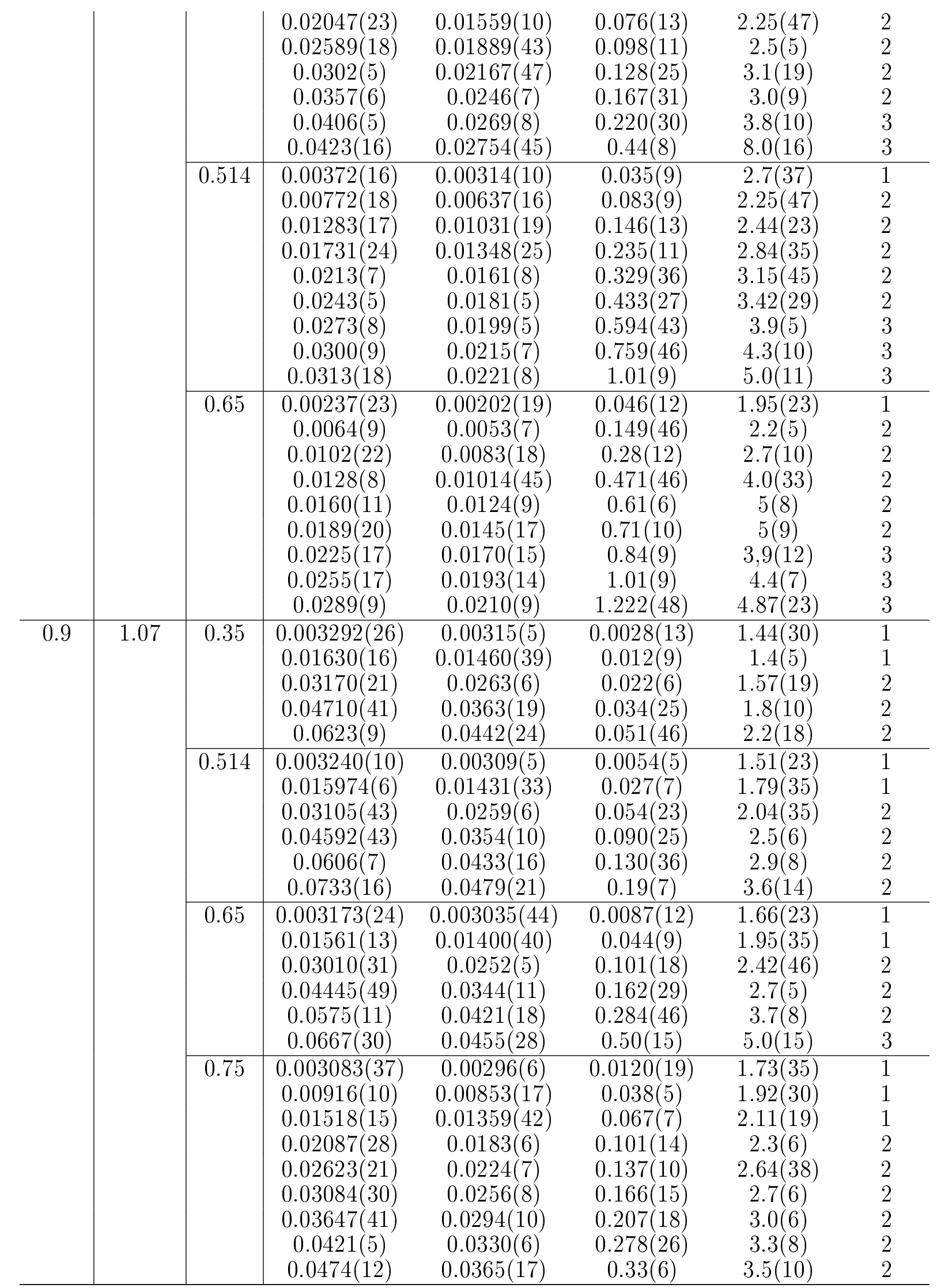




\begin{tabular}{|c|c|c|c|c|c|c|c|}
\hline & & & $\begin{array}{c}0.0522(8) \\
0.0627(34)\end{array}$ & $\begin{array}{l}0.0390(10) \\
0.0443(20)\end{array}$ & $\begin{array}{c}0.414(32) \\
0.70(16)\end{array}$ & $\begin{array}{l}3.7(10) \\
5.0(14)\end{array}$ & $\begin{array}{l}2 \\
3 \\
\end{array}$ \\
\hline \multirow[t]{48}{*}{1.1} & \multirow[t]{36}{*}{1.07} & \multirow[t]{4}{*}{0.35} & $0.003311(23)$ & $0.00388(6)$ & $0.0019(11)$ & $1.32(19)$ & 1 \\
\hline & & & $0.03173(10)$ & $0.0339(10)$ & $0.0166(48)$ & $1.26(19)$ & 2 \\
\hline & & & $0.06245(33)$ & $0.0609(23)$ & $0.029(19)$ & $1.57(46)$ & 2 \\
\hline & & & $0.0909(7)$ & $0.0804(33)$ & $0.053(5)$ & $1.82(23)$ & 2 \\
\hline & & \multirow[t]{5}{*}{0.514} & $0.003273(21)$ & $0.00384(6)$ & $0.0036(11)$ & $1.48(19)$ & 1 \\
\hline & & & $0.03135(12)$ & $0.03356(26)$ & $0.034(5)$ & $1.76(30)$ & 1 \\
\hline & & & $0.0616(6)$ & $0.0605(25)$ & $0.067(29)$ & $2.1(6)$ & 2 \\
\hline & & & $0.0898(7)$ & $0.0801(23)$ & $0.097(45)$ & $2.3(9)$ & 2 \\
\hline & & & $0.1210(8)$ & $0.098(6)$ & $0.116(34)$ & $2.4(9)$ & 2 \\
\hline & & \multirow[t]{6}{*}{0.65} & $0.003232(26)$ & $0.00379(6)$ & $0.0057(13)$ & $1.57(35)$ & 1 \\
\hline & & & $0.03090(11)^{\prime}$ & $0.03305(43)$ & $0.056(5)$ & $1.98(23)$ & 2 \\
\hline & & & $0.0606(6)$ & $0.0597(18)$ & $0.115(24)$ & $2.5(6)$ & 2 \\
\hline & & & $0.0883(7)$ & $0.0787(21)$ & $0.17(5)$ & $2.9(12)$ & 2 \\
\hline & & & $0.1190(9)$ & $0.0961(44)$ & $0.212(44)$ & $2.95(38)$ & 2 \\
\hline & & & $0.1492(24)$ & $0.1108(47)$ & $0.28(13)$ & $3.9(37)$ & 3 \\
\hline & & \multirow[t]{7}{*}{1.0} & $0.003050(23)$ & $0.003596(26)$ & $0.0146(12)$ & $1.63(19)$ & 1 \\
\hline & & & $0.02881(25)$ & $0.03104(38)$ & $0.158(13)^{\prime}$ & $2.36(35)$ & 2 \\
\hline & & & $0.0560(5)$ & $0.0556(20)$ & $0.341(28)$ & $2.95(38)$ & 2 \\
\hline & & & $0.0823(7)$ & $0.0754(35)$ & $0.461(39)$ & $3.4(6)$ & 2 \\
\hline & & & $0.1113(16)$ & $0.092(5)$ & $0.59(8)$ & $3.9(8)$ & 3 \\
\hline & & & $0.1403(16)$ & $0.1062(23)$ & $0.70(6)$ & $4.6(9)$ & 3 \\
\hline & & & $0.1713(21)$ & $0.119(6)$ & $0.75(8)$ & $4.8(10)$ & 3 \\
\hline & & \multirow[t]{7}{*}{1.5} & $0.00238(16)$ & $0.00281(18)$ & $0.048(8)$ & $1.92(30)$ & 1 \\
\hline & & & $0.02224(18)$ & $0.0245(7)$ & $0.484(9)$ & $2.95(38)$ & 2 \\
\hline & & & $0.0478(7)$ & $0.0490(25)$ & $0.744(33)$ & $3.71(48)$ & 2 \\
\hline & & & $0.0740(13)$ & $0.0695(30)$ & $0.87(7)$ & $4.0(7)$ & 3 \\
\hline & & & $0.1029(14)$ & $0.0886(42)$ & $0.99(6)$ & $4.6(11)$ & 3 \\
\hline & & & $0.1324(17)$ & $0.1028(41)$ & $1.07(9)$ & $4.97(48)$ & 3 \\
\hline & & & $0.1624(28)$ & $0.116(5)$ & $1.17(16)$ & $5.9(30)$ & 4 \\
\hline & & \multirow[t]{7}{*}{2.0} & $0.0013(6)$ & $0.0015(7)$ & $0.104(28)$ & $2.2(6)$ & 1 \\
\hline & & & $0.01706(43)$ & 0.0191(9) & $0.743(21)$ & $3.02(30)$ & 2 \\
\hline & & & $0.0434(10)^{\prime}$ & $0.0449(23)$ & $0.96(5)$ & $3.80468(0)$ & 3 \\
\hline & & & $0.0697(7)$ & $0.0661(32)$ & $1.079(39)$ & $4.09(46)$ & 3 \\
\hline & & & $0.0985(13)$ & $0.086(6)$ & $1.21(8)$ & $4.7(7)$ & 3 \\
\hline & & & $0.1281(18)$ & $0.1030(10)$ & $1.277(41)$ & $5.06(30)$ & 3 \\
\hline & & & $0.1582(18)$ & $0.1142(27)$ & $1.36(14)$ & $5.9(14)$ & 4 \\
\hline & \multirow[t]{12}{*}{2.1} & \multirow[t]{6}{*}{0.35} & $0.00324(6)$ & $0.00380(13)$ & $0.0024(31)$ & $1.34(37)$ & 1 \\
\hline & & & $0.03093(33)$ & $0.0333(8)$ & $0.025(18)$ & $1.5(9)$ & 2 \\
\hline & & & $0.06192(49)$ & $0.0616(17)$ & $0.050(32)$ & $1.9(5)$ & 2 \\
\hline & & & $0.0934(7)$ & $0.0831(46)$ & $0.057(32)$ & $1.6(9)$ & 2 \\
\hline & & & $0.1241(10)$ & $0.101(9)$ & $0.08(6)$ & $1.9(15)$ & 3 \\
\hline & & & $0.1568(15)$ & $0.116(9)$ & $0.10(11)$ & $2.7(19)$ & 3 \\
\hline & & \multirow[t]{6}{*}{0.514} & $0.00318(5)$ & $0.00375(12)$ & $0.0051(29)$ & $1.46(46)$ & 1 \\
\hline & & & $0.03035(32)$ & $0.0332(10)^{\prime}$ & $0.055(18)^{\prime}$ & $1.93(46)$ & 2 \\
\hline & & & $0.0606(5)$ & $0.0604(26)$ & $0.116(35)$ & $2.4(11)$ & 2 \\
\hline & & & $0.0915(11)$ & $0.0815(5)$ & $0.16(6)$ & $2.6(10)$ & 2 \\
\hline & & & $0.1215(8)$ & $0.099(8)$ & $0.21(9)$ & $3.0(12)$ & 3 \\
\hline & & & $0.1533(15)$ & $0.114(9)$ & $0.27(11)$ & $3.8(18)$ & 3 \\
\hline
\end{tabular}




\begin{tabular}{|c|c|c|c|c|c|c|c|}
\hline & & 0.65 & $\begin{array}{c}0.00311(6) \\
0.02958(30) \\
0.0590(7) \\
0.0889(9) \\
0.1184(6) \\
0.1490(20)\end{array}$ & $\begin{array}{c}0.00366(13) \\
0.0318(6) \\
0.0583(24) \\
0.080(6) \\
0.098(10) \\
0.113(9)\end{array}$ & $\begin{array}{c}0.0089(30) \\
0.093(16) \\
0.197(39) \\
0.29(6) \\
0.37(5) \\
0.47(12)\end{array}$ & $\begin{array}{c}1.59(42) \\
2.08(46) \\
2.7(6) \\
3.0(7) \\
3.39(37) \\
5(7)\end{array}$ & $\begin{array}{l}1 \\
2 \\
2 \\
2 \\
3 \\
3\end{array}$ \\
\hline & 3.98 & 0.35 & $0.00316(7)$ & $0.00371(10)$ & $0.0053(37)$ & $1.6(10)$ & 1 \\
\hline & & & $0.02979(45)$ & $0.0320(10)$ & $0.042(24)$ & $1.88(41)$ & 2 \\
\hline & & & $0.0588(6)$ & $0.0575(23)$ & $0.07(5)$ & $2.0(8)$ & 2 \\
\hline & & & $0.0883(13)$ & $0.079(5)$ & $0.097(64)$ & $2.07(35)$ & 2 \\
\hline & & & $0.1176(19)$ & $0.0960(41)$ & $0.16(11)$ & $2.8(14)$ & 3 \\
\hline & & & $0.1481(21)$ & $0.109(6)$ & $0.16(13)$ & $2,3(29)$ & 3 \\
\hline & & 0.514 & $0.00306(11)$ & $0.00359(15)$ & $0.010(6)$ & $1.8(14)$ & 1 \\
\hline & & & $0.0286(7)$ & $0.0308(11)^{\prime}$ & $0.103(36)$ & $2.2(7)$ & 2 \\
\hline & & & $0.0562(16)$ & $0.0556(35)$ & $0.20(10)$ & $2.7(16)$ & 2 \\
\hline & & & $0.0848(7)$ & $0.0763(43)$ & $0.285(42)$ & $2.9(5)$ & 2 \\
\hline & & & $0.1129(24)$ & $0.095(6)$ & $0.40(8)$ & $3.8(14)$ & 3 \\
\hline & & & $0.1422(31)$ & $0.108(6)$ & $0.46(15)$ & $4.2(35)$ & 3 \\
\hline & & 0.65 & $0.00290(11)$ & $0.00341(14)$ & $0.019(6)$ & $1.8(9)$ & 1 \\
\hline & & & $0.0269(9)$ & $0.0292(17)^{\prime}$ & $0.191(46)$ & $2.7(21)$ & 2 \\
\hline & & & $0.0532(7)$ & $0.0537(21)$ & $0.355(43)$ & $3.1(7)$ & 2 \\
\hline & & & $0.0808(19)$ & $0.0737(24)$ & $0.49(9)$ & $3.4(9)$ & 3 \\
\hline & & & $0.1088(25)$ & $0.092(8)$ & $0.60(9)$ & $4.1(10)$ & 3 \\
\hline & & & $0.1372(30)$ & $0.1050(45)$ & $0.72(23)$ & $4.9(25)$ & 3 \\
\hline 1.5 & 1.07 & 0.35 & $0.003321(11)$ & $0.00534(8)$ & $0.0013(6)$ & & \\
\hline & & & $0.03182(14)$ & $0.0488(11)$ & $0.010(6)$ & & \\
\hline & & & $0.06254(27)$ & $0.0924(35)$ & $0.017(13)$ & & \\
\hline & & & $0.09108(20)$ & $0.129(7)$ & $0.027(10)$ & & \\
\hline & & 0.514 & $0.003300(23)$ & $0.00531(7)$ & $0.0022(11)$ & & \\
\hline & & & $0.03164(16)$ & $0.0487(17)$ & $0.018(8)$ & & \\
\hline & & & $0.06218(17)$ & 0.0921(38) & $0.035(19)$ & & \\
\hline & & & $0.09051(39)$ & $0.128(5)$ & $0.048(13)$ & & \\
\hline & & 0.65 & $0.003279(22)$ & $0.00528(9)$ & $0.0032(11)$ & & \\
\hline & & & $0.03143(11)^{\prime}$ & $0.0482(11)$ & $0.028(6)$ & & \\
\hline & & & $0.06177(32)$ & $0.0914(41)$ & $0.052(17)$ & & \\
\hline & & & $0.0899(6)$ & $0.1281(44)$ & $0.073(37)$ & & \\
\hline & & & $0.1212(7)$ & $0.168(7)$ & $0.077(21)$ & & \\
\hline 2.0 & 1.07 & 0.35 & $0.003325(14)$ & $0.00716(8)$ & $0.0009(7)$ & & \\
\hline & & & $0.03182(13)$ & $0.0672(12)$ & $0.008(7)$ & & \\
\hline & & & $0.06254(18)$ & $0.131(6)$ & $0.011(10)$ & & \\
\hline & & & $0.0911(5)$ & $0.190(5)$ & $0.013(5)$ & & \\
\hline & & 0.514 & $0.003314(8)$ & $0.00713(9)$ & $0.00145(46)$ & & \\
\hline & & & $0.03172(7)$ & $0.0671(12)$ & $0.0127(39)$ & & \\
\hline & & & $0.06231(19)$ & $0.130(5)$ & $0.021(6)$ & & \\
\hline & & & $0.09076(39)$ & $0.188(9)$ & $0.027(19)$ & & \\
\hline & & & $0.1219(6)$ & $0.253(12)$ & $0.031(22)$ & & \\
\hline & & 0.65 & $0.003301(15)$ & $0.00710(9)$ & $0.0020(8)$ & & \\
\hline & & & $0.03161(15)$ & $0.0666(12)$ & $0.018(7)$ & & \\
\hline & & & $0.06211(26)$ & $0.130(6)$ & $0.031(12)$ & & \\
\hline & & & $0.09033(35)$ & $0.186(7)$ & $0.043(20)$ & & \\
\hline
\end{tabular}




\begin{tabular}{|c|c|c|c|c|c|}
\hline & & & $0.1215(6)$ & $0.252(16)$ & $0.046(13)$ \\
\hline \multirow[t]{49}{*}{3.0} & \multirow[t]{15}{*}{1.07} & 0.35 & $0.003325(11)$ & $0.01079(10)$ & $0.0006(5)$ \\
\hline & & & $0.03182(8)$ & $0.1033(31)$ & $0.0047(40)$ \\
\hline & & & $0.06244(14)$ & $0.205(8)$ & $0.008(9)$ \\
\hline & & & $0.09076(6)$ & $0.302(17)$ & $0.009(10)$ \\
\hline & & & $0.12192(48)$ & $0.417(17)$ & $0.008(11)$ \\
\hline & & 0.514 & $0.003321(14)$ & $0.01076(15)$ & $0.0008(7)$ \\
\hline & & & $0.03178(7)$ & $0.1034(22)$ & $0.0069(32)$ \\
\hline & & & $0.06233(11)$ & $0.203(8)$ & $0.013(6)$ \\
\hline & & & $0.09067(15)$ & $0.301(12)$ & $0.017(6)$ \\
\hline & & & $0.12170(48)$ & $0.416(19)$ & $0.019(15)$ \\
\hline & & 0.65 & $0.003315(12)$ & $0.01073(12)$ & $0.0011(6)$ \\
\hline & & & $0.03172(9)$ & $0.1031(25)^{\prime}$ & $0.0099(47)$ \\
\hline & & & $0.06216(13)$ & $0.203(6)$ & $0.020(6)$ \\
\hline & & & $0.09050(7)$ & $0.302(13)$ & $0.024(7)$ \\
\hline & & & $0.12150(18)$ & $0.412(19)$ & $0.027(5)$ \\
\hline & \multirow[t]{21}{*}{2.1} & 0.35 & $0.003258(47)$ & $0.01054(19)$ & $0.0008(26)$ \\
\hline & & & $0.01548(15)$ & $0.0504(13)$ & $0.006(8)$ \\
\hline & & & $0.03111(20)$ & $0.1018(25)$ & $0.011(11)$ \\
\hline & & & $0.04702(27)$ & $0.1509(32)$ & $0.009(15)$ \\
\hline & & & $0.06231(24)$ & $0.202(12)$ & $0.010(13)$ \\
\hline & & & $0.09359(35)$ & $0.314(19)$ & $0.012(20)$ \\
\hline & & & $0.12418(26)$ & $0.418(23)$ & $0.013(33)$ \\
\hline & & 0.514 & $0.00325(5)$ & $0.01050(15)$ & $0.0012(31)$ \\
\hline & & & $0.01543(15)$ & $0.0505(14)^{\prime}$ & $0.008(8)$ \\
\hline & & & $0.03102(23)$ & $0.1016(27)$ & $0.015(13)$ \\
\hline & & & $0.04690(28)$ & $0.1509(40)$ & $0.014(15)$ \\
\hline & & & $0.06216(24)$ & $0.203(12)$ & $0.018(14)$ \\
\hline & & & $0.09337(34)$ & $0.314(19)$ & $0.024(20)$ \\
\hline & & & $0.12387(29)$ & $0.415(25)$ & $0.029(33)$ \\
\hline & & 0.65 & $0.003241(47)$ & $0.01048(21)$ & $0.0017(27)$ \\
\hline & & & $0.01541(18)$ & $0.0503(16)$ & $0.009(10)$ \\
\hline & & & $0.03094(25)$ & $0.1009(29)$ & $0.019(14)$ \\
\hline & & & $0.04680(37)$ & $0.1505(47)$ & $0.019(20)$ \\
\hline & & & $0.06201(24)$ & $0.203(12)$ & $0.025(13)$ \\
\hline & & & $0.09318(35)$ & $0.314(19)$ & $0.032(21)$ \\
\hline & & & $0.1237(6)$ & $0.417(22)$ & $0.038(37)$ \\
\hline & \multirow[t]{13}{*}{3.98} & 0.35 & $0.003228(35)$ & $0.010458(30)$ & $0.0013(19)$ \\
\hline & & & $0.01549(7)$ & $0.05052(48)$ & $0.0047(41)$ \\
\hline & & & $0.03030(17)$ & $0.0978(18)$ & $0.010(10)$ \\
\hline & & & $0.04593(28)$ & $0.1497(41)$ & $0.014(17)$ \\
\hline & & & $0.05938(46)$ & $0.196(12)$ & $0.022(23)$ \\
\hline & & & $0.08934(20)$ & $0.293(12)$ & $0.013(22)$ \\
\hline & & & $0.1188(7)$ & $0.400(14)$ & $0.021(31)$ \\
\hline & & 0.514 & $0.003210(44)$ & $0.01039(16)$ & $0.0022(25)$ \\
\hline & & & $0.01543(11)$ & $0.0502(9)$ & $0.008(7)$ \\
\hline & & & $0.03017(24)$ & $0.0974(20)$ & $0.017(14)$ \\
\hline & & & $0.04577(35)$ & $0.149(6)$ & $0.022(19)$ \\
\hline & & & $0.05910(49)$ & $0.195(11)$ & $0.033(27)$ \\
\hline & & & $0.08892(49)$ & $0.292(13)$ & $0.031(31)$ \\
\hline
\end{tabular}




\begin{tabular}{|l|c|ccc} 
& & $0.1184(6)$ & $0.397(21)$ & $0.040(31)$ \\
\cline { 4 - 5 } & 0.65 & $0.00320(6)$ & $0.01033(20)$ & $0.0028(33)$ \\
& & $0.01537(6)$ & $0.0500(7)$ & $0.011(5)$ \\
& & $0.03006(26)$ & $0.0971(21)$ & $0.022(15)$ \\
& & $0.04562(22)$ & $0.148(5)$ & $0.029(13)$ \\
& & $0.0589(5)$ & $0.194(11)$ & $0.045(26)$ \\
& & $0.08862(37)$ & $0.291(12)$ & $0.044(24)$ \\
& & $0.1118(6)$ & $0.397(21)$ & $0.056(31)$ \\
\hline
\end{tabular}

In the main article already all adsorption isotherms are shown. However, only selected adsorption layer thickness curves for $T / T_{\mathrm{c}}=0.8$ and $T / T_{\mathrm{c}}=1.1$. In Figure 2,3 , and 4 the remaining ones are shown.

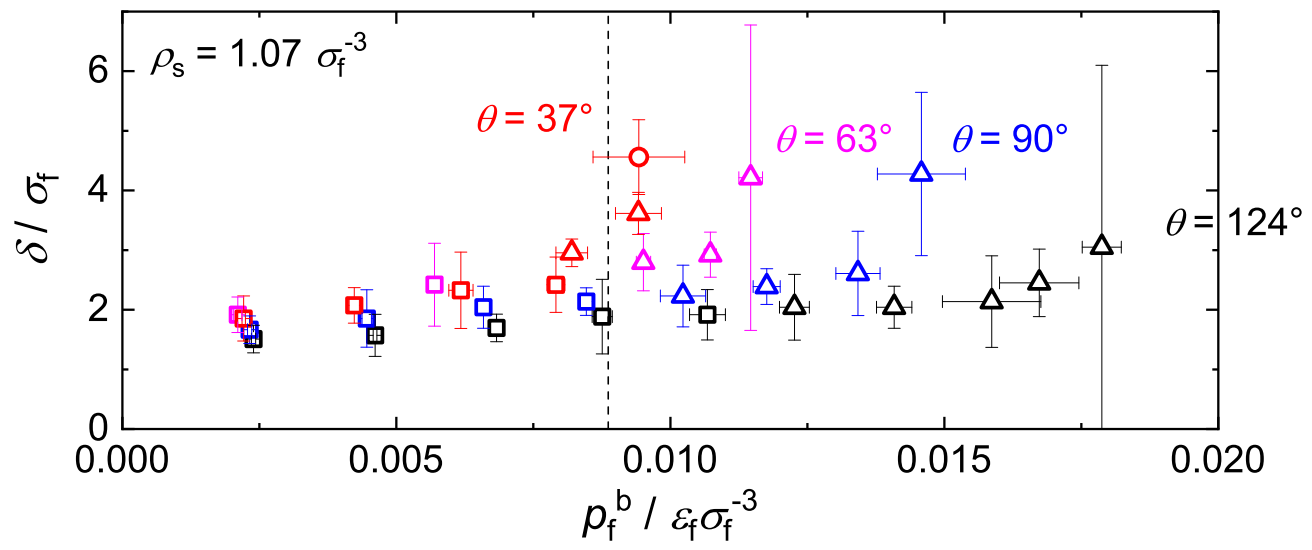

Figure 2: Adsorption layer thickness for $T / T_{\mathrm{c}}=0.7$ and $\rho_{\mathrm{s}}=1.07 \sigma_{\mathrm{f}}^{-3}$. The simulation data is shown as symbols, the uncertainty is indicated by error bars. The number of peaks in the adsorption layer is shown with different symbols: one peak $(\square)$, two peaks $(\triangle)$, and three peaks $(\bigcirc)$. The saturation pressure is shown as a black dashed line and the contact angle $\theta$ is reported for all $W . W=1.08$ (black), $W=1.58$ (blue), $W=2.00$ (violet), and $W=2.31$ (red).

As already mentioned in the article, the curvature of the layer thickness is the same than the curvature of the adsorption isotherms. For $T / T_{\mathrm{c}}=0.77$ a jump in the layer thickness at the wetting transition occurs. The results, discussed in the article, also apply to the results shown here. 


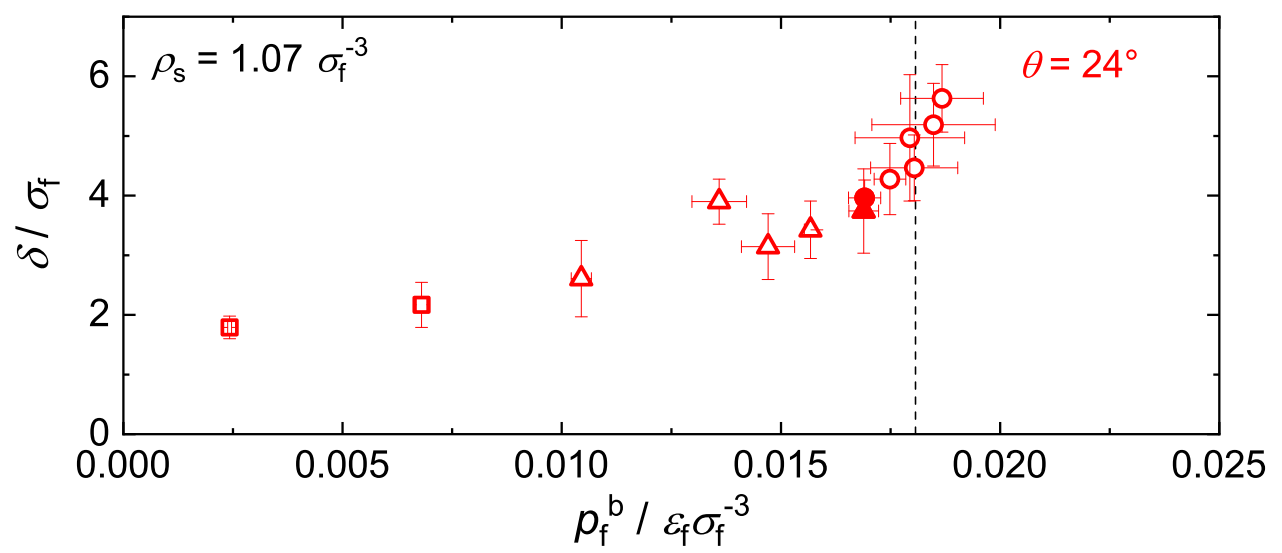

Figure 3: Adsorption layer thickness for $T / T_{\mathrm{c}}=0.77$ and $\rho_{\mathrm{s}}=1.07 \sigma_{\mathrm{f}}^{-3}$. The simulation data is shown as symbols, the uncertainty is indicated by error bars. The number of peaks in the adsorption layer is shown with different symbols: one peak ( $\square)$, two peaks $(\triangle)$, and three peaks $(\bigcirc)$. The saturation pressure is shown as a black dashed line and the contact angle $\theta$ is reported for $W=2.31$ (red). The filled symbols show a jump in the layer thickness where prewetting occurs.

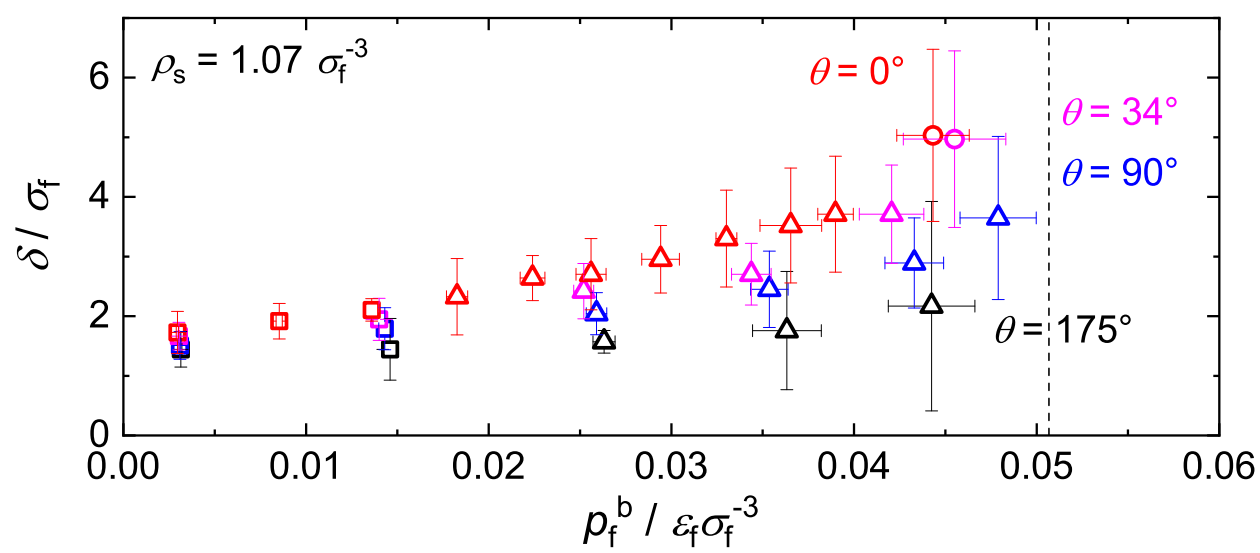

Figure 4: Adsorption layer thickness for $T / T_{\mathrm{c}}=0.9$ and $\rho_{\mathrm{s}}=1.07 \sigma_{\mathrm{f}}^{-3}$. The simulation data is shown as symbols, the uncertainty is indicated by error bars. The number of peaks in the adsorption layer is shown with different symbols: one peak $(\square)$, two peaks $(\triangle)$, and three peaks $(\bigcirc)$. The saturation pressure is shown as a black dashed line and the contact angle $\theta$ is reported for all $W . W=1.08$ (black), $W=1.58$ (blue), $W=2.00$ (violet), and $W=2.31$ (red). 


\section{Detection of Droplet Growth in the Metastable Region}

For an increasing supersaturation of the vapor phase a droplet growth at the surface was

observed. This is indicated by a decrease in the bulk fluid pressure and a droplet-like layering in a density field averaged in $z$-direction $\rho_{\mathrm{f}}(x, y)$. Figure 5 shows such a density field with droplet growth.

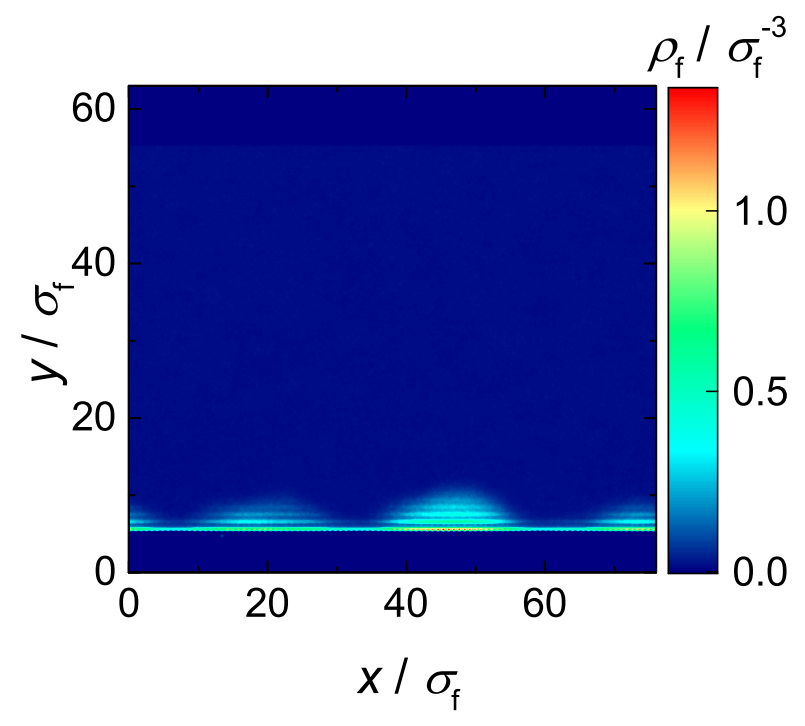

Figure 5: Density field $\rho_{\mathrm{f}}(y, x)$ averaged in $z$-direction.

The colors show the values of the fluid density. The first adsorption layer on the wall is continuous whereas the higher layers only occur in some areas. This indicates a droplet growth. When a decrease in the bulk fluid pressure or a droplet growth in the density field $\rho_{\mathrm{f}}(x, y)$ is observed, no adsorption is calculated.

\section{PeTS Equation of State}

The PeTS Equation of State (EOS) describes the thermodynamic behavior of the LJTS fluid with a cutoff radius of $2.5 \sigma_{\mathrm{f}}{ }^{1}$ In the following, the equations for the PeTS EOS are provided. In this section all physical properties are reduced using the potential parameters 
$\varepsilon_{\mathrm{f}}$ and $\sigma_{\mathrm{f}}$, cf. Table 4 .

Table 4: Overview of physical properties in reduced units.

\begin{tabular}{c|c}
\hline length & $\tilde{z}=z / \sigma_{\mathrm{f}}$ \\
temperature & $\tilde{T}=k_{\mathrm{B}} T / \varepsilon_{\mathrm{f}}$ \\
density & $\tilde{\rho}=\rho \sigma_{\mathrm{f}}^{3}$ \\
free energy per particle & $\tilde{a}=a / \varepsilon_{\mathrm{f}}$ \\
energy & $\tilde{u}=u / \varepsilon_{\mathrm{f}}$ \\
\hline
\end{tabular}

The PeTS EOS is based on the perturbation theory of Barker and Henderson ${ }^{2}$ which means that the residual free energy $\tilde{a}^{\text {res }}$ is split into a free energy of a reference contribution $\tilde{a}^{\text {ref }}$ and a perturbation contribution $\tilde{a}^{\text {pert }}$

$$
\tilde{a}^{\mathrm{res}} \equiv \tilde{a}-\tilde{a}^{\mathrm{id}}=\tilde{a}^{\mathrm{ref}}+\tilde{a}^{\mathrm{pert}}
$$

In equation (3) $\tilde{a}$ is the free energy per particle and $\tilde{a}^{\text {id }}$ is the free energy per particle of the ideal gas. The LJTS potential is discretised with the modified step-square-well potential proposed by Chen and Kreglewski ${ }^{3}$ which leads to two parameters $c_{1}$ and $c_{2} \cdot{ }^{1}$ The approach of Barker and Henderson ${ }^{2}$ leads to the effective hard-shpere diameter $\tilde{d}$ :

$$
\tilde{d}(T)=\int_{0}^{1}\left(1-\exp \left(-\frac{\tilde{u}(\tilde{r})}{\tilde{T}}\right)\right) \mathrm{d} \tilde{r} .
$$

In addition with the step-square-well potential this yields

$$
\tilde{d}(\tilde{T})=1-c_{1} \exp \left(-\frac{c_{2}}{\tilde{T}}\right)
$$

The reduced packing fraction is described in dependency of $\tilde{d}^{4,5}$

$$
\eta=\frac{\pi}{6} \tilde{\rho} \tilde{d}^{3}
$$

where $\tilde{\rho}$ is the number density. Using this approach, the reference term in equation (3) is 
the free energy of the hard-sphere

$$
\frac{\tilde{a}^{\mathrm{ref}}}{\tilde{T}}=\frac{\tilde{a}^{\mathrm{hs}}}{\tilde{T}}=\frac{3 \eta}{1-\eta}+\frac{\eta}{(1-\eta)^{2}} .
$$

The perturbation contribution, which describes dispersion, is modeled as ${ }^{2}$

$$
\frac{\tilde{a}^{\text {pert }}}{\tilde{T}}=\frac{\tilde{a}_{1}}{\tilde{T}}+\frac{\tilde{a}_{2}}{\tilde{T}}
$$

where $\tilde{a}_{1}$ is the first order dispersion contribution and $\tilde{a}_{2}$ the second order dispersion contribution

$$
\begin{gathered}
\frac{\tilde{a}_{1}}{\tilde{T}}=-2 \pi \tilde{\rho} \frac{1}{\tilde{T}} \underbrace{\int_{1}^{\infty} \tilde{u}(\tilde{r}) f\left(\frac{\tilde{r}}{\tilde{d}}\right) \tilde{r}^{2} \mathrm{~d} \tilde{r}}_{I_{1}} \\
\frac{\tilde{a}_{2}}{\tilde{T}}=-\pi \tilde{\rho}\left(1+\frac{8 \eta-2 \eta^{2}}{(1-\eta)^{4}}\right)^{-1}\left(\frac{1}{\tilde{T}}\right)^{2} \underbrace{\frac{\partial}{\partial \tilde{\rho}}\left[\int_{1}^{\infty} \tilde{u}(\tilde{r})^{2} f\left(\frac{\tilde{r}}{\tilde{d}}\right) \tilde{r}^{2} \mathrm{~d} \tilde{r}\right]}_{I_{2}} .
\end{gathered}
$$

In equation (9) and (10) $f$ is the segment-segment radial distribution function. In analogy to Gross and Sadowski ${ }^{6}$ the integrals $I_{1}$ and $I_{2}$ are developed into a taylor series as functions of the packing fraction only

$$
\begin{aligned}
& I_{1}=\sum_{i=0}^{6} a_{i} \eta^{i} \\
& I_{2}=\sum_{i=0}^{6} b_{i} \eta^{i}
\end{aligned}
$$

where 14 independent parameters $a_{i}$ and $b_{i}$ with $i \in\{0,1,2,3,4,5,6\}$ are used. Those 14 parameters and $c_{1}$ and $c_{2}$ were fitted to bulk vapor-liquid equilibrium data by Heier et al. ${ }^{1}$ The PeTS EOS also describes states in the metastable region and homogeneous stable states well and it shows no unphysical behavior in the metastable region. Therefore it can be used with density functional theory and density gradient theory. 


\section{References}

(1) Heier, M.; Stephan, S.; Liu, J.; Chapman, W. G.; Hasse, H.; Langenbach, K. Equation of state for the Lennard-Jones truncated and shifted fluid with a cut-off radius of $2.5 \sigma$ based on perturbation theory and its applications to interfacial thermodynamics. Mol. Phys. 2018, 116, 2083-2094.

(2) Barker, J. A.; Henderson, D. Perturbation Theory and Equation of State for Fluids. II. A Successful Theory of Liquids. J. Chem. Phys. 1967, 47, 4714-4721.

(3) Chen, S. S.; Kreglewski, A. Applications of the Augmented van der Waals Theory of Fluids.: I. Pure Fluids. Ber. Bunsen-Ges. Phys. Chem. 1977, 81, 1048-1052.

(4) Boublík, T. Hard-Sphere Equation of State. J. Chem. Phys. 1970, 53, 471-472.

(5) Mansoori, G. A.; Carnahan, N. F.; Starling, K. E.; Leland, T. W. Equilibrium Thermodynamic Properties of the Mixture of Hard Spheres. J. Chem. Phys. 1971, 54, 1523-1525.

(6) Gross, J.; Sadowski, G. Perturbed-Chain SAFT: An Equation of State Based on a Perturbation Theory for Chain Molecules. Ind. Eng. Chem. Res. 2001, 40, 1244-1260. 